\title{
Bounding the number of characters in a block of a finite group
}

\author{
Benjamin Sambale*
}

July 24,2018

\begin{abstract}
We present a strong upper bound on the number $k(B)$ of irreducible characters of a $p$-block $B$ of a finite group $G$ in terms of local invariants. More precisely, the bound depends on a chosen major $B$-subsection $(u, b)$, its normalizer $\mathrm{N}_{G}(\langle u\rangle, b)$ in the fusion system and a weighted sum of the Cartan invariants of $b$. In this way we strengthen and unify previous bounds given by Brauer, Wada, Külshammer-Wada, Héthelyi-Külshammer-Sambale and the present author.
\end{abstract}

Keywords: number of characters in a block, Cartan matrix, Brauer's $k(B)$-Conjecture AMS classification: 20C15, 20C20

\section{Introduction}

Let $B$ be a $p$-block of a finite group $G$ with defect $d$. Since Richard Brauer [4] conjectured that the number of irreducible characters $k(B)$ in $B$ is at most $p^{d}$, there has been great interest in bounding $k(B)$ in terms of local invariants. Brauer and Feit [6] used some properties of the Cartan matrix $C=\left(c_{i j}\right) \in \mathbb{Z}^{l(B) \times l(B)}$ of $B$ to prove their celebrated bound $k(B) \leq p^{2 d}$ (here and in the following $l(B)$ denotes the number of irreducible Brauer characters of $B)$. In the present paper we investigate stronger bounds by making use of further local invariants. By elementary facts on decomposition numbers, it is easy to see that

$$
k(B) \leq \operatorname{tr}(C)
$$

where $\operatorname{tr}(C)$ denotes the trace of $C$. However, it is not true in general that $\operatorname{tr}(C) \leq p^{d}$. In fact, there are examples with $\operatorname{tr}(C)>l(B) p^{d}$ (see [11]) although Brauer already knew that $k(B) \leq l(B) p^{d}$ (see Corollary 15 below) and this was subsequently improved by Olsson [12, Theorem 4]. For this reason, some authors strengthened (11) in a number of ways. Brandt [3, Proposition 4.2] proved

$$
k(B) \leq \operatorname{tr}(C)-l(B)+1
$$

and this was generalized by the present author in [17, Proposition 8] to

$$
k(B) \leq \sum_{i=1}^{m} \operatorname{det}\left(C_{i}\right)-m+1
$$

\footnotetext{
*Fachbereich Mathematik, TU Kaiserslautern, 67653 Kaiserslautern, Germany, sambale@mathematik.uni-kl.de Institut für Mathematik, FSU Jena, 07737 Jena, Germany, benjamin.sambale@uni-jena.de
} 
where $S_{1}, \ldots, S_{m}$ is a partition of $\{1, \ldots, l(B)\}$ and $C_{i}:=\left(c_{s t}\right)_{s, t \in S_{i}}$. Using different methods, Wada [20] observed that

$$
k(B) \leq \operatorname{tr}(C)-\sum_{i=1}^{l(B)-1} c_{i, i+1} .
$$

In Külshammer-Wada [10], the authors noted that (2) is a special case of

$$
k(B) \leq \sum_{1 \leq i \leq j \leq l(B)} q_{i j} c_{i j}
$$

where $q(x)=\sum_{1 \leq i \leq j \leq l(B)} q_{i j} x_{i} x_{j}$ is a (weakly) positive definite integral quadratic form.

Since $C$ is often harder to compute than $k(B)$, it is desirable to replace $C$ by the Cartan matrix of a Brauer correspondent of $B$ in a proper subgroup. For this purpose let $D$ be a defect group of $B$ and choose $u \in \mathrm{Z}(D)$. Then a Brauer correspondent $b$ of $B$ in $\mathrm{C}_{G}(u)$ has defect group $D$ as well. The present author replaced $c_{i j}$ in (3) by the corresponding entries of the Cartan matrix $C_{u}$ of $b$ (see [15, Lemma 1]).

In Héthelyi-Külshammer-Sambale [9, Theorem 2.4] we have invoked Galois actions to obtain stronger bounds although only in the special cases $p=2$ and $l(b)=1$ (see [9, Theorems 3.1 and 4.10]). More precisely, in the latter case we proved

$$
k(B) \leq \sum_{i=1}^{\infty} p^{2 i} k_{i}(B) \leq\left(n+\frac{|\langle u\rangle|-1}{n}\right) \frac{p^{d}}{|\langle u\rangle|} \leq p^{d}=\operatorname{tr}\left(C_{u}\right)
$$

where $n:=\left|\mathrm{N}_{G}(\langle u\rangle, b): \mathrm{C}_{G}(u)\right|$ and $k_{i}(B)$ is the number of irreducible characters of height $i \geq 0$ in $B$. This is a refinement of a result of Robinson [13, Theorem 3.4.3]. In [18, Theorem 2.6], the present author relaxed the condition $l(b)=1$ to the weaker requirement that $\mathcal{N}:=\mathrm{N}_{G}(\langle u\rangle, b) / \mathrm{C}_{G}(u)$ acts trivially on the set $\operatorname{IBr}(b)$ of irreducible Brauer characters of $b$.

In this paper we replace quadratic forms by a matrix $W$ of weights which allows us to drop all restrictions imposed above. We prove the following general result which incorporates the previous special cases (see Section 3 for details).

Theorem A. Let $B$ be a block of a finite group $G$ with defect group $D$. Let $u \in \mathrm{Z}(D)$ and let $b$ be a Brauer correspondent of $B$ in $\mathrm{C}_{G}(u)$. Let $\mathcal{N}:=\mathrm{N}_{G}(\langle u\rangle, b) / \mathrm{C}_{G}(u)$ and let $C$ be the Cartan matrix of the block $\bar{b}$ of $\mathrm{C}_{G}(u) /\langle u\rangle$ dominated by $b$. Let $W \in \mathbb{R}^{l(b) \times l(b)}$ such that $x W x^{t} \geq 1$ for every $x \in \mathbb{Z}^{l(b)} \backslash\{0\}$. Then

$$
k(B) \leq\left(|\mathcal{N}|+\frac{|\langle u\rangle|-1}{|\mathcal{N}|}\right) \operatorname{tr}(W C) \leq|\langle u\rangle| \operatorname{tr}(W C) .
$$

The first inequality is strict if $\mathcal{N}$ acts non-trivially on $\operatorname{IBr}(b)$ and the second inequality is strict if and only if $1<|\mathcal{N}|<|\langle u\rangle|-1$.

In contrast to (44), we cannot replace $k(B)$ by $\sum p^{2 i} k_{i}(B)$ in Theorem $\mathrm{A}$ (the principal 2-block of $\mathrm{SL}(2,3)$ is a counterexample with $u=1)$. By a classical fusion argument of Burnside, the group $\mathcal{N}$ in Theorem $\mathrm{A}$ is induced from the inertial quotient $\mathrm{N}_{G}\left(D, b_{D}\right) / D \mathrm{C}_{G}(D)$ where $b_{D}$ is a Brauer correspondent of $B$ in $\mathrm{C}_{G}(D)$ (see [1, Corollary 4.18]). In particular, $\mathcal{N}$ is a $p^{\prime}$-group and $|\mathcal{N}|$ divides $p-1$.

As noted in previous papers, if $u \in D \backslash \mathrm{Z}(D)$, one still gets upper bounds on the number of height 0 characters and this is of interest with respect to Olsson's Conjecture $k_{0}(B) \leq\left|D: D^{\prime}\right|$ where $D^{\prime}$ denotes the commutator subgroup of $D$. In fact, we will deduce Theorem A from our second main theorem: 
Theorem B. Let $B$ be a block of a finite group $G$ with defect group $D$. Let $u \in D$ and let $b$ be $a$ Brauer correspondent of $B$ in $\mathrm{C}_{G}(u)$. Let $\mathcal{N}:=\mathrm{N}_{G}(\langle u\rangle, b) / \mathrm{C}_{G}(u)$ and let $C$ be the Cartan matrix of the block $\bar{b}$ of $\mathrm{C}_{G}(u) /\langle u\rangle$ dominated by $b$. Let $W \in \mathbb{R}^{l(b) \times l(b)}$ such that $x W x^{t} \geq 1$ for every $x \in \mathbb{Z}^{l(b)} \backslash\{0\}$. Then

$$
k_{0}(B) \leq k_{0}(\langle u\rangle \rtimes \mathcal{N}) \operatorname{tr}(W C) \leq|\langle u\rangle| \operatorname{tr}(W C) .
$$

The first inequality is strict if $\mathcal{N}$ acts non-trivially on $\operatorname{IBr}(b)$.

In the situation of Theorem B we may assume, after conjugation, that $\mathrm{N}_{D}(\langle u\rangle) / \mathrm{C}_{D}(u)$ is a Sylow $p$-subgroup of $\mathcal{N}$ (see [2, Proposition 2.5]). In particular, $\mathcal{N}=\mathrm{N}_{D}(\langle u\rangle) / \mathrm{C}_{D}(u)$ whenever $p=2$.

If $\mathcal{N}$ acts trivially on $\operatorname{IBr}(b)$, then our bounds cannot be improved in general. To see this, let $\langle u\rangle$ be any cyclic $p$-group, and let $\mathcal{N} \leq \operatorname{Aut}(\langle u\rangle)$. Then $G:=\langle u\rangle \rtimes \mathcal{N}$ has only one $p$-block $B$. In this situation $l(b)=1$ and $C=(1)$. Hence, $k_{0}(B)=k_{0}(G)=k_{0}(\langle u\rangle \rtimes \mathcal{N}) \operatorname{tr}(W C)$ for $W=(1)$. Similarly, if $\mathcal{N}$ is a $p^{\prime}$-group, then $k(B)=k(G)=|\mathcal{N}|+\frac{|\langle u\rangle|-1}{|\mathcal{N}|}$.

It is known that the ordinary character table of $\mathrm{C}_{G}(u) /\langle u\rangle$ determines $C$ up to basic sets, i.e. up to transformations of the form $S^{\mathrm{t}} C S$ where $S \in \mathrm{GL}(l(b), \mathbb{Z})$ and $S^{\mathrm{t}}$ is the transpose of $S$. Then $\widetilde{W}:=S^{-1} W S^{-\mathrm{t}}$ still satisfies $x \widetilde{W} x^{\mathrm{t}} \geq 1$ for every $x \in \mathbb{Z}^{l(b)} \backslash\{0\}$ and

$$
\operatorname{tr}\left(\widetilde{W} S^{\mathrm{t}} C S\right)=\operatorname{tr}\left(S^{-1} W C S\right)=\operatorname{tr}(W C) .
$$

Hence, our results do not depend on the chosen basic set.

\section{Proofs}

First we outline the proof of Theorem B: For sake of simplicity suppose first that $u=1$. Then every row $d_{\chi}$ of the decomposition matrix $Q$ of $B$ is non-zero and $Q^{\mathrm{t}} Q=C$. Hence,

$$
k(B) \leq \sum_{\chi \in \operatorname{Irr}(B)} d_{\chi} W d_{\chi}^{\mathrm{t}}=\operatorname{tr}\left(Q W Q^{\mathrm{t}}\right)=\operatorname{tr}\left(W Q^{\mathrm{t}} Q\right)=\operatorname{tr}(W C)
$$

In the general case we replace $Q$ be the generalized decomposition matrix with respect to the subsection $(u, b)$. Then $Q$ consists of algebraic integers in the cyclotomic field of degree $q:=|\langle u\rangle|$. We apply a discrete Fourier transformation to turn $Q$ into an integral matrix with the same number of rows, but with more columns. At the same time we need to blow up $W$ to a larger matrix with similar properties. Afterwards we use the fact that the rows of $Q$ corresponding to height 0 characters are non-zero and fulfill a certain $p$-adic valuation. For $p=2$ the proof can be completed directly, while for $p>2$ we argue by induction on $q$. Additional arguments are required to handle the case where $|\mathcal{N}|$ is divisible by $p$. These calculations make use of sophisticated matrix analysis.

We fix the following matrix notation. For $n \in \mathbb{N}$ let $1_{n}$ be the identity matrix of size $n \times n$ and similarly let $0_{n}$ be the zero matrix of the same size. Moreover, let

$$
U_{n}:=\frac{1}{2}\left(\begin{array}{cccc}
2 & -1 & & 0 \\
-1 & \ddots & \ddots & \\
& \ddots & \ddots & -1 \\
0 & & -1 & 2
\end{array}\right) \in \mathbb{Q}^{n \times n} .
$$

For $d \in \mathbb{N}$ let $d^{n \times n}$ be the $n \times n$ matrix which has every entry equal to $d$. For $A \in \mathbb{R}^{n \times n}$ and $B \in \mathbb{R}^{m \times m}$ we construct the direct sum $A \oplus B \in \mathbb{R}^{(n+m) \times(n+m)}$ and the Kronecker product $A \otimes B \in \mathbb{R}^{n m \times n m}$ 
in the usual manner. Note that $\operatorname{tr}(A \oplus B)=\operatorname{tr}(A)+\operatorname{tr}(B)$ and $\operatorname{tr}(A \otimes B)=\operatorname{tr}(A) \operatorname{tr}(B)$. Finally, let $\delta_{i j}$ be the Kronecker delta. We assume that every positive (semi)definite matrix is symmetric. Moreover, we call a symmetric matrix $A \in \mathbb{R}^{n \times n}$ integral positive definite, if $x A x^{\mathrm{t}} \geq 1$ for every $x \in \mathbb{R}^{n} \backslash\{0\}$.

The proof of Theorem B is deduced from a series of lemmas and propositions.

Lemma 1. Every integral positive definite matrix is positive definite.

Proof. Let $W \in \mathbb{R}^{n \times n}$ be integral positive definite. By way of contradiction, suppose that there exists an eigenvector $v \in \mathbb{R}^{n}$ of $W$ with eigenvalue $\lambda \leq 0$ and (euclidean) norm 1 . If $\lambda<0$, choose $x \in \mathbb{Q}^{n}$ such that $\|x\| \leq\|v\|=1$ and $\|x-v\|<-\frac{\lambda}{2\|W\|}$ where $\|W\|$ denotes the Frobenius matrix norm of $W$. Then

$$
x W x^{\mathrm{t}}=(x-v) W(x+v)^{\mathrm{t}}+v W v^{\mathrm{t}} \leq\|x-v\|\|W\|\|x+v\|+\lambda<0 .
$$

However, there exists $m \in \mathbb{N}$ such that $m x \in \mathbb{Z}^{n}$ and $(m x) W(m x)^{\mathrm{t}}<0$. This contradiction implies $\lambda=0$. By Dirichlet's approximation theorem (see [8, Theorem 200]) there exist infinitely many integers $m$ and $x \in \mathbb{Z}^{n}$ such that

It follows that

$$
\|x-m v\|<\frac{\sqrt{n}}{\sqrt[n]{m}}
$$

$$
x W x^{\mathrm{t}}=(x-m v) W(x-m v)^{\mathrm{t}} \leq\|x-m v\|^{2}\|W\|<1
$$

if $m$ is sufficiently large. Again we have a contradiction.

Conversely, every positive definite matrix can be scaled to an integral positive definite matrix. The next lemma is a key argument when dealing with non-trivial actions of $\mathcal{N}$ on $\operatorname{IBr}(b)$.

Lemma 2. Let $A, B \in \mathbb{R}^{n \times n}$ positive semidefinite matrices such that $A$ commutes with a permutation matrix $P \in \mathbb{R}^{n \times n}$. Then $\operatorname{tr}(A B P) \leq \operatorname{tr}(A B)$. If $A$ and $B$ are positive definite, then $\operatorname{tr}(A B P)=$ $\operatorname{tr}(A B)$ if and only if $P=1_{n}$.

Proof. By the spectral theorem, $A$ and $P$ are diagonalizable. Since they commute, they are simultaneously diagonalizable. Since $A$ has real, non-negative eigenvalues, there exists a positive semidefinite matrix $A^{1 / 2} \in \mathbb{R}^{n \times n}$ such that $A^{1 / 2} A^{1 / 2}=A$ and $A^{1 / 2} P=P A^{1 / 2}$. Then $M:=\left(m_{i j}\right)=A^{1 / 2} B A^{1 / 2}$ is also positive semidefinite. In particular $m_{i j} \leq\left(m_{i i}+m_{j j}\right) / 2$ for $i, j \in\{1, \ldots, n\}$. If $\sigma$ denotes the permutation corresponding to $P$, then we obtain

$$
\operatorname{tr}(A B P)=\operatorname{tr}\left(A^{1 / 2} B P A^{1 / 2}\right)=\operatorname{tr}(M P)=\sum_{i=1}^{n} m_{i \sigma(i)} \leq \sum_{i=1}^{n} \frac{m_{i i}+m_{\sigma(i) \sigma(i)}}{2}=\operatorname{tr}(M)=\operatorname{tr}(A B) .
$$

If $A$ and $B$ are positive definite, then so is $M$ and we have $m_{i j}<\left(m_{i i}+m_{j j}\right) / 2$ whenever $i \neq j$. This implies the last claim.

Lemma 3. Let $W \in \mathbb{R}^{n \times n}$ be integral positive definite and suppose that $W$ commutes with a permutation matrix P. Let

$$
W_{m}:=\frac{1}{2}\left(\begin{array}{cccc}
2 W & -P W & & 0 \\
-P^{t} W & \ddots & \ddots & \\
& \ddots & \ddots & -P W \\
0 & & -P^{t} W & 2 W
\end{array}\right) \in \mathbb{R}^{m n \times m n} .
$$

Then $W_{m}$ is integral positive definite. In particular, $U_{m} \otimes W$ is integral positive definite. 
Proof. Let $x=\left(x_{1}, \ldots, x_{m}\right)$ with $x_{i} \in \mathbb{Z}^{n}$. Since $W P=P W$ we have

$$
\begin{aligned}
x W_{m} x^{\mathrm{t}} & =\sum_{i=1}^{m} x_{i} W x_{i}^{\mathrm{t}}-\sum_{i=1}^{m-1} x_{i} P W x_{i+1}^{\mathrm{t}} \\
& =\frac{1}{2} x_{1} W x_{1}^{\mathrm{t}}+\frac{1}{2} x_{m} W x_{m}^{\mathrm{t}}+\frac{1}{2} \sum_{i=1}^{m-1}\left(x_{i} P-x_{i+1}\right) W\left(x_{i} P-x_{i+1}\right)^{\mathrm{t}} .
\end{aligned}
$$

We may assume that $x_{i} \neq 0$ for some $i \in\{1, \ldots, m\}$. If $i=1$, then $x_{m} \neq 0$ or $x_{j} P \neq x_{j+1}$ for some $j$. In any case $x W_{m} x^{\mathrm{t}} \geq 1$. If $i>1$, then the claim can be seen in a similar fashion. The last claim follows with $P=1_{n}$.

Now assume the notation of Theorem B. In addition, let $p$ be the characteristic of $B$ such that $q:=$ $|\langle u\rangle|$ is a power of $p$. Let $k:=k(B), l:=l(b)$ and $\zeta:=e^{2 \pi i / q} \in \mathbb{C}$. Then the generalized decomposition matrix $Q=\left(d_{\chi \varphi}^{u}\right)$ of $B$ with respect to the subsection $(u, b)$ has size $k \times l$ and entries in $\mathbb{Z}[\zeta]$ (see [16, Definition 1.19] for instance). By the orthogonality relations of generalized decomposition numbers, we have $Q^{\mathrm{t}} \bar{Q}=q C$ where $q C$ is the Cartan matrix of $b$ (see [16, Theorems 1.14 and 1.22]). Recall that $C$ is positive definite and has non-negative integer entries.

The first part of the next lemma is a result of Broue [7] while the second part was known to Brauer [5, $(5 \mathrm{H})]$.

Lemma 4 ([16, Proposition 1.36]). Let $d_{\chi}$ be a row of $Q$ corresponding to a character $\chi \in \operatorname{Irr}(B)$ of height 0 . Let $d$ be the defect of $\bar{b}$ and let $\widetilde{C}:=p^{d} C^{-1} \in \mathbb{Z}^{l \times l}$. Then the $p$-adic valuation of $d_{\chi} \widetilde{C}{\overline{d_{\chi}}}^{t}$ is 0 . In particular, $d_{\chi} \neq 0$. Now assume that $u \in \mathrm{Z}(D)$ and $\chi \in \operatorname{Irr}(B)$ is arbitrary. Then $d_{\chi} \neq 0$.

We identify the Galois group $\mathcal{G}:=\operatorname{Gal}(\mathbb{Q}(\zeta) \mid \mathbb{Q})$ with $\operatorname{Aut}(\langle u\rangle) \cong(\mathbb{Z} / q \mathbb{Z})^{\times}$such that $\gamma(\zeta)=\zeta^{\gamma}$ for $\gamma \in \mathcal{G}$. In this way we regard $\mathcal{N}$ as a subgroup of $\mathcal{G}$. Let $n:=|\mathcal{N}|$. For any $\gamma \in \mathcal{G}, \gamma(Q)$ is the generalized decomposition matrix with respect to $\left(u^{\gamma}, b\right)$. If the subsections $(u, b)$ and $\left(u^{\gamma}, b\right)$ are not conjugate in $G$, then $\gamma \notin \mathcal{N}$ and $\gamma(Q)^{\mathrm{t}} \bar{Q}=0$. On the other hand, if they are conjugate, then $\gamma \in \mathcal{N}$ and

$$
\gamma\left(d_{\chi \varphi}^{u}\right)=d_{\chi \varphi}^{u^{\gamma}}=d_{\chi \varphi^{\gamma}}^{u}
$$

for $\chi \in \operatorname{Irr}(B)$ and $\varphi \in \operatorname{IBr}(b)$. Hence, in this case, $\gamma$ acts on the columns of $Q$ and there exists a permutation matrix $P_{\gamma}$ such that $\gamma(Q)=Q P_{\gamma}$. Recall that permutation matrices are orthogonal, i. e. $P_{\gamma^{-1}}=P_{\gamma}^{-1}=P_{\gamma}^{\mathrm{t}}$. Since $\mathcal{G}$ is abelian, we obtain

$$
C P_{\gamma}=Q^{\mathrm{t}} \gamma(\bar{Q})=\gamma^{-1}(Q)^{\mathrm{t}} \bar{Q}=P_{\gamma^{-1}}^{\mathrm{t}} C=P_{\gamma} C
$$

for every $\gamma \in \mathcal{N}$ and

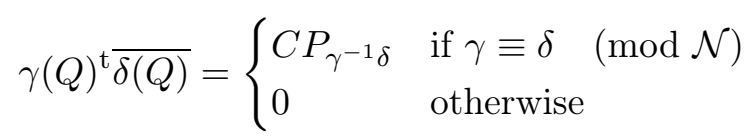

for $\gamma, \delta \in \mathcal{G}$. For any subset $\mathcal{S} \subseteq \mathcal{N}$ we write $P_{\mathcal{S}}:=\sum_{\delta \in \mathcal{S}} P_{\delta}$.

Lemma 5. In the situation of Theorem $B$ we may assume that $W$ is (integral) positive definite and commutes with $P_{\gamma}$ for every $\gamma \in \mathcal{N}$.

Proof. Let

$$
\mathcal{W}:=\frac{1}{2 n} \sum_{\delta \in \mathcal{N}} P_{\delta}\left(W+W^{\mathrm{t}}\right) P_{\delta}^{\mathrm{t}}
$$


Then $\mathcal{W}$ is symmetric and commutes with $P_{\delta}$ for every $\delta \in \mathcal{N}$. Moreover, $\mathcal{W}$ is integral positive definite and by Lemma 1, $\mathcal{W}$ is positive definite. Finally,

$$
\operatorname{tr}(\mathcal{W} C)=\frac{1}{2 n} \sum_{\delta \in \mathcal{N}} \operatorname{tr}\left(P_{\delta} W C P_{\delta}^{\mathrm{t}}\right)+\operatorname{tr}\left(P_{\delta} W^{\mathrm{t}} C P_{\delta}^{\mathrm{t}}\right)=\operatorname{tr}(W C),
$$

since $P_{\delta}$ commutes with $C$. Hence, we may replace $W$ by $\mathcal{W}$.

In the following we revisit some arguments from [16, Section 5.2]. Write $Q=\sum_{i=1}^{\varphi(q)} A_{i} \zeta^{i}$ where $A_{i} \in \mathbb{Z}^{k \times l}$ for $i=1, \ldots, \varphi(q)$ and $\varphi(q)=q-q / p$ is Euler's function. Let

$$
\mathcal{A}_{q}=\left(A_{i}: i=1, \ldots, \varphi(q)\right) \in \mathbb{Z}^{k \times \varphi(q) l} .
$$

Lemma 6. The matrix $\mathcal{A}_{q}$ has rank $l \varphi(q) / n$.

Proof. It is well-known that the Vandermonde matrix $V:=\left(\zeta^{i \gamma}: 1 \leq i \leq \varphi(q), \gamma \in \mathcal{G}\right)$ is invertible. Since $Q$ has full rank, the facts stated above show that $(\gamma(Q): \gamma \in \mathcal{G})$ has rank $l|\mathcal{G}: \mathcal{N}|=l \varphi(q) / n$. Then also $\mathcal{A}_{q}=(\gamma(Q): \gamma \in \mathcal{G})\left(V \otimes 1_{l}\right)^{-1}$ has $\operatorname{rank} l \varphi(q) / n$.

Let $T_{q}$ be the trace of $\mathbb{Q}(\zeta)$ with respect to $\mathbb{Q}$. Recall that

$$
T_{q}\left(\zeta^{i}\right)= \begin{cases}\varphi(q) & \text { if } q \mid i \\ -q / p & \text { if } q \nmid i \text { and } \frac{q}{p} \mid i \\ 0 & \text { otherwise. }\end{cases}
$$

Hence,

$$
T_{q}\left(Q \zeta^{-i}\right)=\sum_{j=1}^{\varphi(q)} A_{j} T_{q}\left(\zeta^{j-i}\right)=\frac{q}{p}\left(p A_{i}-\sum_{j \equiv i} A_{(\bmod q / p)} A_{j} .\right.
$$

Definition 7. For $1 \leq i \leq \varphi(q)$ there is a unique integer $i^{\prime}$ such that $0 \leq i^{\prime}<q / p$ and $i^{\prime} \equiv-i$ $(\bmod q / p)$.

Then $q / p \leq i+i^{\prime} \leq \varphi(q)$ and $\sum_{j \equiv i(\bmod q / p)} \zeta^{-j}=-\zeta^{i^{\prime}}$ where we consider only those summands with $1 \leq j \leq \varphi(q)$. With this convention we obtain

$$
\begin{aligned}
T_{q}\left(Q\left(\zeta^{-i}-\zeta^{i^{\prime}}\right)\right) & =\frac{q}{p}\left(p A_{i}-\sum_{j \equiv i} A_{(\bmod q / p)}+\sum_{j \equiv i}\left(p A_{j}-\sum_{s \equiv j} \sum_{(\bmod q / p)} A_{s}\right)\right) \\
& =\frac{q}{p}\left(p A_{i}+(p-1) \sum_{j \equiv i} A_{j}-(p-1) \sum_{s \equiv i} A_{(\bmod q / p)} A_{s}\right)=q A_{i}
\end{aligned}
$$

and (7) yields

$$
\begin{aligned}
q^{2} A_{i}^{\mathrm{t}} A_{j} & =\sum_{\gamma, \delta \in \mathcal{G}}\left(\zeta^{-i \gamma}-\zeta^{i^{\prime} \gamma}\right)\left(\zeta^{j \delta}-\zeta^{-j^{\prime} \delta}\right) \gamma(Q)^{\mathrm{t}} \delta(\bar{Q}) \\
& =\sum_{\delta \in \mathcal{N}} \sum_{\gamma \in \mathcal{G}}\left(\zeta^{-i \gamma}-\zeta^{i^{\prime} \gamma}\right)\left(\zeta^{j \gamma \delta}-\zeta^{-j^{\prime} \gamma \delta}\right) q C P_{\delta} \\
& =q C \sum_{\delta \in \mathcal{N}} P_{\delta} T_{q}\left(\zeta^{j \delta-i}-\zeta^{j \delta+i^{\prime}}-\zeta^{-j^{\prime} \delta-i}+\zeta^{-j^{\prime} \delta+i^{\prime}}\right)
\end{aligned}
$$


for $1 \leq i, j \leq \varphi(q)$. Note that

$$
j \delta-i \equiv j \delta+i^{\prime} \equiv-j^{\prime} \delta-i \equiv-j^{\prime} \delta+i^{\prime} \quad(\bmod q / p) .
$$

Moreover, if $j \delta-i \equiv 0(\bmod q)$, then $j \delta-i^{\prime} \not \equiv 0(\bmod p)$. In this case $T_{q}\left(\zeta^{j \delta-i}-\zeta^{j \delta+i^{\prime}}\right)=$ $\varphi(q)+q / p=q$. In a similar way we obtain

$$
A_{i}^{\mathrm{t}} A_{j}=C \sum_{\delta \in \mathcal{N}} P_{\delta}\left([j \delta \equiv i]-\left[j \delta \equiv-i^{\prime}\right]+\left[j^{\prime} \delta \equiv i^{\prime}\right]-\left[j^{\prime} \delta \equiv-i\right]\right)
$$

where all congruences are modulo $q$ and [...] denotes the indicator function.

By Lemma 4, $\mathcal{A}_{q}$ has non-zero rows $a_{1}, \ldots, a_{k_{0}(B)}$. If $\mathcal{W} \in \mathbb{R}^{l \varphi(q) \times l \varphi(q)}$ is integral positive definite, then

$$
k_{0}(B) \leq \sum_{i=1}^{k_{0}(B)} a_{i} \mathcal{W} a_{i}^{\mathrm{t}} \leq \operatorname{tr}\left(\mathcal{A}_{q} \mathcal{W} \mathcal{A}_{q}^{\mathrm{t}}\right)=\operatorname{tr}\left(\mathcal{W} \mathcal{A}_{q}^{\mathrm{t}} \mathcal{A}_{q}\right)
$$

and this is what we are going to show. We need to discuss the case $p=2$ separately.

Proposition 8. Theorem B holds for $p=2$.

Proof. If $q \leq 2$, then $Q=A_{1}=\mathcal{A}_{q}, n=1$ and

$$
k_{0}(B) \leq \operatorname{tr}\left(W Q^{\mathrm{t}} Q\right)=\operatorname{tr}(W q C)=q \operatorname{tr}(W C)=k_{0}(\langle u\rangle \rtimes \mathcal{N}) \operatorname{tr}(W C) .
$$

Hence, we will assume for the remainder of the proof that $q \geq 4$. Then $i^{\prime}=q / 2-i$ for every $1 \leq i \leq \varphi(q)=q / 2$. Hence, (8) simplifies to

$$
A_{i}^{\mathrm{t}} A_{j}=2 C \sum_{\delta \in \mathcal{N}} P_{\delta}([j \delta \equiv i]-[j \delta \equiv i+q / 2]) .
$$

It is well-known that

$$
\mathcal{G}=\langle-1+q \mathbb{Z}\rangle \times\langle 5+q \mathbb{Z}\rangle \cong C_{2} \times C_{q / 4}
$$

In particular, $\mathcal{N}$ is a 2-group and so is $U:=\langle u\rangle \rtimes \mathcal{N}$. Therefore, $k_{0}(U)=\left|U: U^{\prime}\right|$ where $U^{\prime}$ denotes the commutator subgroup of $U$.

Case 1: $\mathcal{N}=\left\langle 5^{2^{m}}+q \mathbb{Z}\right\rangle$ for some $m \geq 0$.

Then $q=|\mathcal{N}| 2^{m+2}=n 2^{m+2}$ and $U^{\prime}$ is generated by $u^{5^{2^{m}}-1}$. Since $5^{2^{m}}-1 \equiv 2^{m+2}\left(\bmod 2^{m+3}\right)$, we conclude that $\left|U^{\prime}\right|=n$ and $k_{0}(U)=\left|U: U^{\prime}\right|=q$.

For any given $\delta \in \mathcal{N} \backslash\{1\}$ both congruences $i \delta \equiv i(\bmod q)$ and $i \delta \equiv i+q / 2(\bmod q)$ have solutions $i \in\{1, \ldots, q / 2\}$. Moreover, the number of solutions is the same, since they both form residue classes modulo a common integer. On the other hand, $i \delta \equiv i+q / 2(\bmod q)$ has no solution for $\delta=1$. An application of (9) yields

$$
\sum_{i=1}^{q / 2} A_{i}^{\mathrm{t}} A_{i}=2 C \sum_{\delta \in \mathcal{N}} P_{\delta} \sum_{i=1}^{q / 2}[i \delta \equiv i]-[i \delta \equiv i+q / 2]=q C P_{1}=q C .
$$

The matrix $\mathcal{W}:=1_{q / 2} \otimes W$ is certainly integral positive definite. Moreover,

$$
k_{0}(B) \leq \operatorname{tr}\left(\mathcal{W} \mathcal{A}_{q}^{\mathrm{t}} \mathcal{A}_{q}\right)=\operatorname{tr}\left(\sum_{i=1}^{q / 2} W A_{i}^{\mathrm{t}} A_{i}\right)=q \operatorname{tr}(W C)=k_{0}(U) \operatorname{tr}(W C) .
$$


It remains to check when this bound is sharp. If $k_{0}(B)=\operatorname{tr}\left(\mathcal{W} \mathcal{A}_{q}^{\mathrm{t}} \mathcal{A}_{q}\right)$, then every row of $\mathcal{A}_{q}$ vanishes in all but (possibly) one $A_{i}$. Moreover, characters of positive height vanish completely in $\mathcal{A}_{q}$. By way of contradiction, suppose that $\mathcal{N}$ acts non-trivially on $\operatorname{IBr}(b)$. Using (5)), it follows that there exists a character $\chi \in \operatorname{Irr}(B)$ of height 0 such that the corresponding row $d_{\chi}=a \zeta^{i}$ of $Q$ satisfies $a P_{\delta}=-a$ for some $\delta \in \mathcal{N}$. We write $a=\left(\alpha_{1}, \ldots, \alpha_{s},-\alpha_{1}, \ldots,-\alpha_{s}, 0, \ldots, 0\right)$ with non-zero $\alpha_{1}, \ldots, \alpha_{s} \in \mathbb{Z}$. With the notation of Lemma 4 let $\widetilde{C}=\left(\widetilde{c}_{i j}\right)$. By (6), we have $P_{\delta} \widetilde{C}=\widetilde{C} P_{\delta}$. Now Lemma 4 leads to the contradiction

$$
0 \not \equiv d_{\chi} \widetilde{C}{\overline{d_{\chi}}}^{\mathrm{t}}=a \widetilde{C} a^{\mathrm{t}} \equiv \sum_{i=1}^{s} 2 \alpha_{i}^{2} \widetilde{c}_{i i} \equiv 0 \quad(\bmod 2),
$$

since the diagonal of $\widetilde{C}$ is constant on the orbits of $\mathcal{N}$. Therefore, equality in (10) can only hold if $\mathcal{N}$ acts trivially on $\operatorname{IBr}(b)$.

Case 2: $\delta:=-5^{m}+q \mathbb{Z} \in \mathcal{N} \backslash\{1\}$ for some $m \geq 0$.

Since $1+5^{m} \equiv 2(\bmod 4)$, we have $U^{\prime}=\left\langle u^{1+5^{m}}\right\rangle=\left\langle u^{2}\right\rangle$ and $k_{0}(U)=\left|U: U^{\prime}\right|=2 n$. We show that every row of $A_{q / 2}$ corresponding to a height 0 character $\chi \in \operatorname{Irr}(B)$ is non-zero. Let $d_{\chi}=\sum_{i=1}^{q / 2} a_{i} \zeta^{i}$ be the corresponding row of $Q$ where $a_{i}$ is a row of $A_{i}$. Let $\nu$ be the $p$-adic valuation. By Lemma 4 ,

$$
0=\nu\left(d_{\chi} \widetilde{C}{\overline{d_{\chi}}}^{\mathrm{t}}\right)=\nu\left(\sum_{1 \leq i, j \leq q / 2} a_{i} \widetilde{C} a_{j}^{\mathrm{t}} \zeta^{i-j}\right)=\nu\left(\sum_{i=1}^{q / 2} a_{i} \widetilde{C} a_{i}^{\mathrm{t}}\right),
$$

i. e.

$$
\sum_{i=1}^{q / 2} a_{i} \widetilde{C} a_{i}^{\mathrm{t}} \equiv 1 \quad(\bmod 2)
$$

On the other hand,

$$
\sum_{i=1}^{q / 2} a_{i} P_{\delta} \zeta^{i}=d_{\chi} P_{\delta}=\delta\left(d_{\chi}\right)=\sum_{i=1}^{q / 2} a_{i} \zeta^{i \delta} .
$$

Now $i \delta \equiv i(\bmod q)$ implies $-5^{m} \equiv \delta \equiv 1(\bmod q / \operatorname{gcd}(q, i))$ and $i=q / 2$. Similarly $i \delta \equiv i+q / 2$ $(\bmod q)$ implies $i=q / 4$. Then $A_{q / 4} P_{\delta}=-A_{q / 4}$. As in Case 1, it follows that $a_{q / 4} \widetilde{C} a_{q / 4}^{\mathrm{t}} \equiv 0(\bmod 2)$. For $i \notin\{q / 2, q / 4\}$ we have $A_{i} P_{\delta}= \pm A_{j}$ for some $j \in\{1, \ldots, q / 2\} \backslash\{i\}$. Then, using (6) ,

$$
a_{j} \widetilde{C} a_{j}^{\mathrm{t}}=a_{i} P_{\delta} \widetilde{C} P_{\delta}^{\mathrm{t}} a_{i}^{\mathrm{t}}=a_{i} \widetilde{C} a_{i}^{\mathrm{t}} .
$$

Now (111) yields $a_{q / 2} \widetilde{C} a_{q / 2}^{\mathrm{t}} \equiv 1(\bmod 2)$ and $a_{q / 2} \neq 0$. Therefore, $A_{q / 2}$ has non-zero rows for height 0 characters.

By (9), $A_{d / 2}^{\mathrm{t}} A_{d / 2}=2 C P_{\mathcal{N}}$ and Lemma 2 implies

$$
k_{0}(B) \leq \operatorname{tr}\left(W A_{d / 2}^{\mathrm{t}} A_{d / 2}\right)=2 \operatorname{tr}\left(W C P_{\mathcal{N}}\right)=2 \sum_{\gamma \in \mathcal{N}} \operatorname{tr}\left(W C P_{\gamma}\right) \leq 2 n \operatorname{tr}(W C)=k_{0}(U) \operatorname{tr}(W C)
$$

with strict inequality if $\mathcal{N}$ acts non-trivially on $\operatorname{IBr}(b)$.

We are left with the case $p>2$. Here $\mathcal{G}$ is cyclic and $\mathcal{N}$ is uniquely determined by $n$. Let $n_{p}$ be the $p$-part of $n$ and $n_{p^{\prime}}$ the $p^{\prime}$-part. Then $n_{p} \mid \frac{q}{p}$ and $n_{p^{\prime}} \mid p-1$.

Lemma 9. We have $k_{0}(\langle u\rangle \rtimes \mathcal{N})=n+\frac{q-n_{p}}{n_{p^{\prime}}}$ for $p>2$. 
Proof. The inflations from $\mathcal{N}$ yield $n$ linear characters in $U:=\langle u\rangle \rtimes \mathcal{N}$, since $\mathcal{N}$ is cyclic. Now let $1 \neq \lambda \in \operatorname{Irr}(\langle u\rangle)$. If the orbit size of $\lambda$ under $\mathcal{N}$ is divisible by $p$, then the irreducible characters of $U$ lying over $\lambda$ all have positive height. Hence, we may assume that $\lambda^{q / n_{p}}=1$. Then, by Clifford theory, $\lambda$ extends in $n_{p}$ many ways to $\langle u\rangle \rtimes \mathcal{N}_{p}$ where $\mathcal{N}_{p}$ is the Sylow $p$-subgroup of $\mathcal{N}$. All these extensions induce to irreducible characters of $U$ of height 0 . We have $\frac{q / n_{p}-1}{n_{p^{\prime}}}$ choices for $\lambda$. Thus, in total we obtain

$$
k_{0}(U)=n+n_{p} \frac{q / n_{p}-1}{n_{p^{\prime}}}=n+\frac{q-n_{p}}{n_{p^{\prime}}} .
$$

The following settles Theorem B in the special case $n_{p}=1$ (use Lemma 9).

Proposition 10. Let $p>2$ and $n_{p}=1$. With the notation above there exists an integral positive definite matrix $\mathcal{W} \in \mathbb{R}^{\varphi(q) l \times \varphi(q) l}$ such that

$$
\operatorname{tr}\left(\mathcal{W} \mathcal{A}_{q}^{t} \mathcal{A}_{q}\right) \leq\left(n+\frac{q-1}{n}\right) \operatorname{tr}(W C)
$$

with equality if and only if $\mathcal{N}$ acts trivially on $\operatorname{IBr}(b)$.

Proof. We argue by induction on $q$. If $q=1$, then $\mathcal{A}_{q}=A_{1}=Q, n=1$ and the claim holds with $\mathcal{W}=W($ Lemma 5). The next case requires special treatment as well.

Case 1: $q=p$.

Then $i^{\prime}=0$ for all $i$ and (8) simplifies to

$$
A_{i}^{\mathrm{t}} A_{j}=C \sum_{\delta \in \mathcal{N}} P_{\delta}([j \delta \equiv i]+[0 \delta \equiv 0])=\left\{\begin{array}{lll}
C P_{\mathcal{N}} & \text { if } i \not j j & (\bmod \mathcal{N}) \\
C\left(P_{\mathcal{N}}+P_{j^{-1} i}\right) & \text { if } i \equiv j & (\bmod \mathcal{N})
\end{array}\right.
$$

After permuting the columns of $\mathcal{A}_{q}$ if necessary, we obtain

$$
\mathcal{A}_{q}^{\mathrm{t}} \mathcal{A}_{q}=1^{\varphi(q) \times \varphi(q)} \otimes P_{\mathcal{N}} C+1_{n^{\prime}} \otimes\left(P_{\gamma^{-1} \delta} C\right)_{\gamma, \delta \in \mathcal{N}}
$$

where $n^{\prime}:=(p-1) / n$. We fix a generator $\rho$ of $\mathcal{N}$. Then we may write $\left(P_{\gamma^{-1} \delta} C\right)_{\gamma, \delta \in \mathcal{N}}=\left(P_{\rho}^{j-i} C\right)_{i, j=1}^{n}$. By Lemma 5, we may assume that $W$ is (integral) positive definite and commutes with $P_{\rho}$. Let $W_{n}$ as in Lemma 3 where we use $P_{\rho}$ instead of $P$. A repeated application of that lemma shows that the matrix $\mathcal{W}:=U_{n^{\prime}} \otimes W_{n}$ is integral positive definite. Moreover, since $P_{\mathcal{N}} P_{\rho}=P_{\mathcal{N}}=P_{\mathcal{N}} P_{\rho}^{\mathrm{t}}$, we have

$$
\begin{aligned}
\operatorname{tr}\left(\mathcal{W} \mathcal{A}_{q}^{\mathrm{t}} \mathcal{A}_{q}\right) & =\operatorname{tr}\left(\left(U_{n^{\prime}} \otimes W_{n}\right)\left(1^{\varphi(q) \times \varphi(q)} \otimes P_{\mathcal{N}} C\right)\right)+\operatorname{tr}\left(\left(U_{n^{\prime}} \otimes W_{n}\right)\left(1_{n^{\prime}} \otimes\left(P_{\rho}^{j-i} C\right)\right)\right) \\
& =\operatorname{tr}\left(\left(U_{n^{\prime}} \otimes W_{n}\right)\left(1^{n^{\prime} \times n^{\prime}} \otimes 1^{n \times n} \otimes P_{\mathcal{N}} C\right)\right)+\operatorname{tr}\left(U_{n^{\prime}} \otimes W_{n}\left(P_{\rho}^{j-i} C\right)\right) \\
& =\operatorname{tr}\left(U_{n^{\prime}} 1^{n^{\prime} \times n^{\prime}}\right) \operatorname{tr}\left(W_{n}\left(1^{n \times n} \otimes P_{\mathcal{N}} C\right)\right)+\operatorname{tr}\left(U_{n^{\prime}}\right) \operatorname{tr}\left(W_{n}\left(P_{\rho}^{j-i} C\right)\right) \\
& =\operatorname{tr}\left(W_{n}\left(1^{n \times n} \otimes P_{\mathcal{N}} C\right)\right)+n^{\prime} \operatorname{tr}\left(W_{n}\left(P_{\rho}^{j-i} C\right)\right) \\
& =\sum_{i=1}^{n} \operatorname{tr}\left(W C P_{\mathcal{N}}\right)-\sum_{i=1}^{n-1} \operatorname{tr}\left(W C P_{\mathcal{N}} P_{\rho}\right)+n^{\prime}\left(\sum_{i=1}^{n} \operatorname{tr}(W C)-\sum_{i=1}^{n-1} \operatorname{tr}\left(W C P_{\rho} P_{\rho}^{\mathrm{t}}\right)\right) \\
& =\operatorname{tr}\left(W C P_{\mathcal{N}}\right)+n^{\prime} \operatorname{tr}(W C) .
\end{aligned}
$$

Finally, Lemma 2 implies

$$
\operatorname{tr}\left(W C P_{\mathcal{N}}\right)=\sum_{\delta \in \mathcal{N}} \operatorname{tr}\left(W C P_{\delta}\right) \leq n \operatorname{tr}(W C)
$$


with equality if and only if $\mathcal{N}$ acts trivially on $\operatorname{IBr}(b)$. This completes the proof in the case $q=p$.

Case 2: $q>p$.

Let

$$
I_{p}:=\{1 \leq i \leq \varphi(q): p \mid i\}, \quad I_{p^{\prime}}:=\{1 \leq i \leq \varphi(q): p \nmid i\} .
$$

Then $\left|I_{p}\right|=\varphi(q) / p=\varphi(q / p)$ and $\left|I_{p^{\prime}}\right|=\varphi(q)-\varphi(q / p)=\varphi(q / p)(p-1)$. If $i \in I_{p}$ and $j \in I_{p^{\prime}}$, then $j \delta-i \not \equiv 0(\bmod q / p)$ for every $\delta \in \mathcal{N}$ and $A_{i}^{\mathrm{t}} A_{j}=0$ by (8). Hence, after relabeling the columns of $\mathcal{A}_{q}$, we obtain

$$
\mathcal{A}_{q}^{\mathrm{t}} \mathcal{A}_{q}=\left(\begin{array}{cc}
\Delta_{p} & 0 \\
0 & \Delta_{p^{\prime}}
\end{array}\right)
$$

where $\Delta_{p}$ corresponds to the indices in $I_{p}$. Since $n \mid p-1$, we may regard $\mathcal{N}$ as a subgroup of $\operatorname{Gal}\left(\mathbb{Q}\left(\zeta^{p}\right) \mid \mathbb{Q}\right)$. For $i \in I_{p}$ let $j=i / p$. Then $i^{\prime} \equiv-i(\bmod q / p)$ implies $i^{\prime} / p \equiv-j\left(\bmod q / p^{2}\right)$ and $0 \leq i^{\prime} / p<q / p^{2}$. Hence, $j^{\prime}=i^{\prime} / p$ where the left hand side refers to $q / p$. It follows from (8) that $\Delta_{p}=\mathcal{A}_{q / p}^{\mathrm{t}} \mathcal{A}_{q / p}$. By induction on $q$ there exists an integral positive definite $\mathcal{W}_{p}$ such that

$$
\operatorname{tr}\left(\mathcal{W}_{p} \Delta_{p}\right) \leq\left(n+\frac{q / p-1}{n}\right) \operatorname{tr}(W C)
$$

with equality if and only if $\mathcal{N}$ acts trivially on $\operatorname{IBr}(b)$.

It remains to consider $\Delta_{p^{\prime}}$. By Lemma $6, \mathcal{A}_{q / p}$ and $\Delta_{p}$ have rank $l \varphi(q / p) / n$ and therefore $\Delta_{p^{\prime}}$ has rank

$$
l(\varphi(q)-\varphi(q / p)) / n=l \varphi(q / p)(p-1) / n .
$$

We define a subset $J \subseteq I_{p^{\prime}}$ such that $|J|=\varphi(q / p)(p-1) / n$ and the matrix $\left(A_{i}: i \in J\right)$ has full rank. Let $R$ be a set of representatives for the orbits of $\left\{i \in I_{p^{\prime}}: 1 \leq i \leq q / p\right\}$ under the multiplication action of $\mathcal{N}$ modulo $q / p$. Note that every orbit has size $n$. For $r \in R$ let

$$
J_{r}:=\{r+j q / p: j=0, \ldots, p-2\} \subseteq I_{p^{\prime}}
$$

and $J:=\bigcup_{r \in R} J_{r}$. Since $J_{r} \cap J_{s}=\varnothing$ for $r \neq s$, we have $|J|=\varphi(q / p)(p-1) / n$. If $i \in J_{r}$ and $j \in J_{s}$ with $r \neq s$, then $j \delta \not \equiv i(\bmod q / p)$ for every $\delta \in \mathcal{N}$. Consequently, $A_{i}^{\mathrm{t}} A_{j}=0$. Now let $i, j \in J_{r}$. Then (8) implies

$$
A_{i}^{\mathrm{t}} A_{j}=C\left(1+\delta_{i j}\right)
$$

After relabeling we obtain

$$
\left(A_{i}: i \in J\right)^{\mathrm{t}}\left(A_{i}: i \in J\right)=1_{\varphi(q / p) / n} \otimes\left(1+\delta_{i j}\right)_{i, j=1}^{p-1} \otimes C .
$$

In particular, $\left(A_{i}: i \in J\right)$ has full rank. Since $\Delta_{p^{\prime}}$ has the same rank, there exists an integral matrix $S \in \operatorname{GL}(l \varphi(q / p)(p-1), \mathbb{Q})$ such that

$$
S^{\mathrm{t}} \Delta_{p^{\prime}} S=1_{\varphi(q / p) / n} \otimes\left(1+\delta_{i j}\right) \otimes C \oplus 0_{s}
$$

where $s:=l \varphi(q / p)(p-1)(n-1) / n$. Let

$$
\mathcal{W}_{p^{\prime}}:=S\left(1_{\varphi(q / p) / n} \otimes U_{p-1} \otimes W \oplus 1_{s}\right) S^{\mathrm{t}} .
$$

Then $\mathcal{W}_{p^{\prime}}$ is integral positive definite by Lemma 3 , Moreover,

$$
\begin{aligned}
\operatorname{tr}\left(\mathcal{W}_{p^{\prime}} \Delta_{p^{\prime}}\right) & =\operatorname{tr}\left(\left(1_{\varphi(q / p) / n} \otimes U_{p-1} \otimes W\right)\left(1_{\varphi(q / p) / n} \otimes\left(1+\delta_{i j}\right) \otimes C\right)\right)+\operatorname{tr}\left(1_{s} 0_{s}\right) \\
& =\frac{\varphi(q / p)}{n} \operatorname{tr}\left(U_{p-1}\left(1+\delta_{i j}\right)\right) \operatorname{tr}(W C)=\frac{\varphi(q / p) p}{n} \operatorname{tr}(W C)=\frac{\varphi(q)}{n} \operatorname{tr}(W C) .
\end{aligned}
$$


Finally, we set $\mathcal{W}:=\mathcal{W}_{p} \oplus \mathcal{W}_{p^{\prime}}$. Then $\mathcal{W}$ is integral positive definite and

$$
\begin{aligned}
\operatorname{tr}\left(\mathcal{W} \mathcal{A}_{q}^{\mathrm{t}} \mathcal{A}_{q}\right) & =\operatorname{tr}\left(\mathcal{W}_{p} \Delta_{p}\right)+\operatorname{tr}\left(\mathcal{W}_{p^{\prime}} \Delta_{p^{\prime}}\right) \leq\left(n+\frac{q / p-1}{n}\right) \operatorname{tr}(W C)+\frac{\varphi(q)}{n} \operatorname{tr}(W C) \\
& =\left(n+\frac{q-1}{n}\right) \operatorname{tr}(W C)
\end{aligned}
$$

with equality if and only if $\mathcal{N}$ acts trivially on $\operatorname{IBr}(b)$.

To complete the proof of Theorem B it remains to show the following.

Proposition 11. Theorem $B$ holds in the case $p>2$ and $n_{p}>1$.

Proof. Let

$$
I_{1}:=\left\{1 \leq i \leq \varphi(q): n_{p} \mid i\right\}, \quad I_{2}:=\left\{1 \leq i \leq \varphi(q): n_{p} \nmid i\right\} .
$$

As in the proof of Proposition 10 we have

$$
\mathcal{A}_{q}^{\mathrm{t}} \mathcal{A}_{q}=\left(\begin{array}{cc}
\Delta_{1} & 0 \\
0 & \Delta_{2}
\end{array}\right)
$$

where $\Delta_{1}$ corresponds to the indices in $I_{1}$. Let $\mathcal{N}=\mathcal{N}_{p} \times \mathcal{N}_{p^{\prime}}$ where $\mathcal{N}_{p}:=\left\langle 1+q / n_{p}+q \mathbb{Z}\right\rangle$ is the unique Sylow $p$-subgroup of $\mathcal{N}$. Then $\delta i \equiv i(\bmod q)$ for $\delta \in \mathcal{N}_{p}$ and $i \in I_{1}$. Hence, for $i, j \in I_{1}$ we have

$$
\begin{aligned}
A_{i}^{\mathrm{t}} A_{j} & =C \sum_{\delta \in \mathcal{N}} P_{\delta}\left([j \delta \equiv i]-\left[j \delta \equiv-i^{\prime}\right]+\left[j^{\prime} \delta \equiv i^{\prime}\right]-\left[j^{\prime} \delta \equiv-i\right]\right) \\
& =C P_{\mathcal{N}_{p}} \sum_{\delta \in \mathcal{N}_{p^{\prime}}} P_{\delta}\left([j \delta \equiv i]-\left[j \delta \equiv-i^{\prime}\right]+\left[j^{\prime} \delta \equiv i^{\prime}\right]-\left[j^{\prime} \delta \equiv-i\right]\right) .
\end{aligned}
$$

For $i \in I_{1}$ it is easy to see that $i^{\prime} / n_{p}=\left(i / n_{p}\right)^{\prime}$ when the right hand side is considered with respect to $q / n_{p}$ (see proof of Proposition 10). It follows that

$$
\Delta_{1}=\left(1_{\varphi\left(q / n_{p}\right)} \otimes P_{\mathcal{N}_{p}}\right) \mathcal{A}_{q / n_{p}}^{\mathrm{t}} \mathcal{A}_{q / n_{p}}
$$

where we consider $\mathcal{A}_{q / n_{p}}$ with respect to the $p^{\prime}$-group $\mathcal{N}_{p^{\prime}}$. By Proposition 10, there exists an integral positive definite $\mathcal{W}_{1}$ such that

$$
\operatorname{tr}\left(\mathcal{W}_{1} \mathcal{A}_{q / n_{p}}^{\mathrm{t}} \mathcal{A}_{q / n_{p}}\right) \leq\left(n_{p^{\prime}}+\frac{q / n_{p}-1}{n_{p^{\prime}}}\right) \operatorname{tr}(W C)
$$

Moreover, equality holds if and only if $\mathcal{N}_{p^{\prime}}$ acts trivially on $\operatorname{IBr}(b)$. By construction, $\mathcal{A}_{q / n_{p}}^{\mathrm{t}} \mathcal{A}_{q / n_{p}}$ is positive semidefinite. By (6) and ([8), $\mathcal{A}_{q / n_{p}}^{\mathrm{t}} \mathcal{A}_{q / n_{p}}$ commutes with $1_{\varphi\left(q / n_{p}\right)} \otimes P_{\delta}$ for $\delta \in \mathcal{N}_{p}$. Hence, Lemma 2 implies

$$
\begin{aligned}
\operatorname{tr}\left(\mathcal{W}_{1} \Delta_{1}\right) & =\operatorname{tr}\left(\mathcal{W}_{1}\left(1_{\varphi\left(q / n_{p}\right)} \otimes P_{\mathcal{N}_{p}}\right) \mathcal{A}_{q / n_{p}}^{\mathrm{t}} \mathcal{A}_{q / n_{p}}\right) \\
& \leq n_{p} \operatorname{tr}\left(\mathcal{W}_{1} \mathcal{A}_{q / n_{p}}^{\mathrm{t}} \mathcal{A}_{q / n_{p}}\right) \leq\left(n+\frac{q-n_{p}}{n_{p^{\prime}}}\right) \operatorname{tr}(W C)
\end{aligned}
$$

Suppose that $\operatorname{tr}\left(\mathcal{W}_{1} \Delta_{1}\right)=\left(n+\frac{q-n_{p}}{n_{p^{\prime}}}\right) \operatorname{tr}(W C)$. Then, by (12), $\mathcal{N}_{p^{\prime}}$ acts trivially on $\operatorname{IBr}(b)$ and the matrices $A_{i}^{\mathrm{t}} A_{j}$ with $i, j \in I_{1}$ are scalar multiples of $C P_{\mathcal{N}_{p}}$. We write $\mathcal{A}_{q / n_{p}}^{\mathrm{t}} \mathcal{A}_{q / n_{p}}=\left(A_{i j}\right)$ such that 
$A_{i n_{p}}^{\mathrm{t}} A_{j n_{p}}=P_{\mathcal{N}_{p}} A_{i j}$. Note that $A_{11}=2 C$ is positive definite. As in the proof of Lemma 2, we construct a positive semidefinite matrix $M=\left(m_{i j}\right):=A^{1 / 2} \mathcal{W}_{1} A^{1 / 2}$ where $A^{1 / 2} A^{1 / 2}=\left(A_{i j}\right)_{i, j}$. By way of contradiction, suppose that $P_{\delta} \neq 1_{l}$ for some $\delta \in \mathcal{N}_{p}$. Let $1 \leq i \leq l$ such that $\delta(i) \neq i$, and let $x=\left(x_{j}\right) \in \mathbb{Z}^{\varphi\left(q / n_{p}\right) l}$ with $x_{i}=-x_{\delta(i)}=1$ and zero elsewhere. Then $x\left(A_{i j}\right) x^{\mathrm{t}}>0$ since $A_{11}$ is positive definite. Thus, $A^{1 / 2} x^{\mathrm{t}} \neq 0$. Since $\mathcal{W}_{1}$ is positive definite (Lemma 1), it follows that $x M x^{\mathrm{t}}>0$ and $m_{i \delta(i)}<\left(m_{i i}+m_{\delta(i) \delta(i)}\right) / 2$. Hence, the proof of Lemma 2 leads to

$$
\begin{aligned}
\operatorname{tr}\left(\mathcal{W}_{1}\left(1_{\varphi\left(q / n_{p}\right)} \otimes P_{\delta}\right) \mathcal{A}_{q / n_{p}}^{\mathrm{t}} \mathcal{A}_{q / n_{p}}\right) & =\operatorname{tr}\left(A^{1 / 2} \mathcal{W}_{1}\left(1_{\varphi\left(q / n_{p}\right)} \otimes P_{\delta}\right) A^{1 / 2}\right) \\
& =\operatorname{tr}\left(M\left(1_{\varphi\left(q / n_{p}\right)} \otimes P_{\delta}\right)\right)<\operatorname{tr}(M)=\operatorname{tr}\left(\mathcal{W}_{1} \mathcal{A}_{q / n_{p}}^{\mathrm{t}} \mathcal{A}_{q / n_{p}}\right)
\end{aligned}
$$

and we derive the contradiction $\operatorname{tr}\left(\mathcal{W}_{1} \Delta_{1}\right)<n_{p} \operatorname{tr}\left(\mathcal{W}_{1} \mathcal{A}_{q / n_{p}}^{\mathrm{t}} \mathcal{A}_{q / n_{p}}\right)$. Thus, we have shown that equality in (13) can only hold if $\mathcal{N}$ acts trivially on $\operatorname{IBr}(b)$.

Now we use the argument from Proposition 8 to deal with $\Delta_{2}$. Let $\chi \in \operatorname{Irr}(B)$ of height 0 , and let $d_{\chi}=\sum_{i=1}^{\varphi(q)} a_{i} \zeta^{i}$ be the corresponding row of $Q$. By Lemma 4, we have

$$
0=\nu\left(d_{\chi} \widetilde{C}{\overline{d_{\chi}}}^{\mathrm{t}}\right)=\nu\left(\sum_{i, j=1}^{\varphi(q)} a_{i} \widetilde{C} a_{j}^{\mathrm{t}}\right)
$$

where $\nu$ is the $p$-adic valuation. In order to show that $a_{i} \neq 0$ for some $i \in I_{1}$, it suffices to show that

$$
\sum_{i, j \in I_{2}} a_{i} \widetilde{C} a_{j}^{\mathrm{t}} \equiv 0 \quad(\bmod p)
$$

For any $\delta \in \mathcal{N}_{p}$ we have

$$
\sum_{i=1}^{\varphi(q)} A_{i} P_{\delta} \zeta^{i}=Q P_{\delta}=\delta(Q)=\sum_{i=1}^{\varphi(q)} A_{i} \zeta^{i \delta} .
$$

Restricting to the indices $i \in I_{2}$ and taking the valuation yields

$$
\sum_{i \in I_{2}} A_{i} P_{\delta} \equiv \sum_{i \in I_{2}} A_{i} \quad(\bmod p)
$$

Let $i \in I_{2}$ be arbitrary and choose $\delta \in \mathcal{N}_{p}$ such that $\operatorname{gcd}(q, i) p=|\langle\delta\rangle|$. Let

$$
\left\{i_{1}, \ldots, i_{p-1}\right\}=\left\{j \in I_{2}: j \equiv i \quad(\bmod q / p)\right\} .
$$

We may assume that $i_{1} \delta \equiv-i^{\prime}(\bmod q)$ and $i_{j} \delta \equiv i_{j-1}(\bmod q)$ for $j=2, \ldots, p-1$. Since $\zeta^{-i^{\prime}}=$ $-\zeta^{i_{1}}-\ldots-\zeta^{i_{p-1}}$, we obtain $A_{i_{p-1}} P_{\delta}=-A_{i_{1}}$ and $A_{i_{j}} P_{\delta}=A_{i_{j+1}}-A_{i_{1}}$ for $j=1, \ldots, p-2$. Hence,

$$
\begin{aligned}
\left(\sum_{j \in I_{2}} a_{j}\right) \widetilde{C} a_{i_{1}}^{\mathrm{t}} & =\left(\sum_{j \in I_{2}} a_{j}\right) P_{\delta} \widetilde{C} P_{\delta}^{\mathrm{t}} a_{i_{1}}^{\mathrm{t}} \equiv\left(\sum_{j \in I_{2}} a_{j}\right) \widetilde{C}\left(a_{i_{2}}-a_{i_{1}}\right)^{\mathrm{t}} \equiv\left(\sum_{j \in I_{2}} a_{j}\right) \widetilde{C}\left(a_{i_{3}}-a_{i_{2}}\right)^{\mathrm{t}} \\
& \equiv \ldots \equiv\left(\sum_{j \in I_{2}} a_{j}\right) \widetilde{C}\left(a_{i_{p-1}}-a_{i_{p-2}}\right)^{\mathrm{t}} \equiv-\left(\sum_{j \in I_{2}} a_{j}\right) \widetilde{C} a_{i_{p-1}}^{\mathrm{t}}(\bmod p) .
\end{aligned}
$$

Now it is easy to see that

$$
\left(\sum_{j \in I_{2}} a_{j}\right) \widetilde{C}\left(a_{i_{1}}+\ldots+a_{i_{p-1}}\right)^{\mathrm{t}} \equiv \frac{p(p-1)}{2}\left(\sum_{j \in I_{2}} a_{j}\right) \widetilde{C} a_{i_{1}}^{\mathrm{t}} \equiv 0 \quad(\bmod p)
$$


and (14) follows. Thus, we have shown that every height 0 character has a non-vanishing part in $A_{i}$ for some $i \in I_{1}$. Hence by (13),

$$
k_{0}(B) \leq \operatorname{tr}\left(\mathcal{W}_{1} \Delta_{1}\right) \leq\left(n+\frac{q-n_{p}}{n_{p^{\prime}}}\right) \operatorname{tr}(W C)
$$

with strict inequality if $\mathcal{N}$ acts non-trivially on $\operatorname{IBr}(b)$. By Lemma 9, the proof is complete.

Now it is time to derive Theorem A from Theorem B. For the convenience of the reader we restate it as follows.

Proposition 12. If $u \in \mathrm{Z}(D)$ in the situation above, then

$$
k(B) \leq\left(n+\frac{q-1}{n}\right) \operatorname{tr}(W C) \leq q \operatorname{tr}(W C) .
$$

The first inequality is strict if $\mathcal{N}$ acts non-trivially on $\operatorname{IBr}(b)$ and the second inequality is strict if and only if $1<n<q-1$.

Proof. As mentioned in the introduction, $\mathcal{N}$ is induced from the inertial quotient $\mathrm{N}_{G}\left(D, b_{D}\right) / D \mathrm{C}_{G}(D)$ and therefore $\mathcal{N}$ is a $p^{\prime}$-group. As a subgroup of $\operatorname{Aut}(\langle u\rangle)$, its order $n$ must divide $p-1$. For $p=2$ we obtain $n=1$ and $k_{0}(\langle u\rangle \rtimes \mathcal{N})=q$. For $p>2$, Lemma 9 gives $k_{0}(\langle u\rangle \rtimes \mathcal{N})=n+\frac{q-1}{n}$. By Lemma 4, all rows of $Q$ are non-zero. Hence, the proofs of Propositions 8 and 10 actually show that $k(B) \leq k_{0}(\langle u\rangle \rtimes \mathcal{N}) \operatorname{tr}(W C)$ with strict inequality if $\mathcal{N}$ acts non-trivially on $\operatorname{IBr}(b)$ (note that only Case 1 in the proof of Proposition 8 is relevant). This implies the first two claims. The last claim follows, since $n+\frac{q-1}{n}$ is a convex function in $n$ and $1 \leq n \leq q-1$.

If the action of $\mathcal{N}$ on $\operatorname{IBr}(b)$ is known, a careful analysis of the proofs above leads to even stronger estimates. For instance, in Proposition 10 we have actually shown that

$$
k_{0}(B) \leq \operatorname{tr}\left(W C P_{\mathcal{N}}\right)+\frac{q-1}{n} \operatorname{tr}(W C)
$$

for $p>2$ and $n_{p}=1$. If $\bar{b}$ has cyclic defect groups, then $P_{\mathcal{N}}$ is a direct sum of equal blocks of the form $d^{n / d \times n / d}$ (see [18, Proposition 3.2]). This can be used to give a simpler proof of [18, Theorem 3.1].

\section{Consequences}

In this section we deduce some of the results stated in the introduction.

Corollary 13 (Sambale [14, Lemma 1]). Let $C=\left(c_{i j}\right)_{i, j=1}^{l}$ be the Cartan matrix of a Brauer correspondent of $B$ in $\mathrm{C}_{G}(u)$ where $u \in \mathrm{Z}(D)$. Then for every positive definite, integral quadratic form $q\left(x_{1}, \ldots, x_{l}\right)=\sum_{1 \leq i \leq j \leq l} q_{i j} x_{i} x_{j}$ we have

$$
k(B) \leq \sum_{1 \leq i \leq j \leq l} q_{i j} c_{i j}
$$


Proof. Let $t:=|\langle u\rangle|$. Then $t^{-1} C$ is the Cartan matrix of the block $\bar{b}$ in Theorem A (see [16, Theorem 1.22]). Taking $W:=\frac{1}{2}\left(q_{i j}\left(1+\delta_{i j}\right)\right)$ with $q_{i j}=q_{j i}$ we obtain

$$
x W x^{\mathrm{t}}=\frac{1}{2} \sum_{1 \leq i, j \leq l} q_{i j}\left(1+\delta_{i j}\right) x_{i} x_{j}=\sum_{1 \leq i \leq j \leq l} q_{i j} x_{i} x_{j}=q(x) \geq 1
$$

for every $x=\left(x_{1}, \ldots, x_{l}\right) \in \mathbb{Z}^{l} \backslash\{0\}$ and

$$
k(B) \leq t \operatorname{tr}\left(W t^{-1} C\right)=\operatorname{tr}(W C)=\sum_{1 \leq i \leq j \leq l} q_{i j} c_{i j} .
$$

Wada's inequality (2) follows from Corollary 13 with $q(x)=\sum_{i=1}^{l} x_{i}^{2}-\sum_{i=1}^{l-1} x_{i} x_{i+1}$ (or $W=U_{l}$ in Theorem A).

Corollary 14 (Héthelyi-Külshammer-Sambale [9, Theorem 4.10]). Suppose $p>2$. Let b be a Brauer correspondent of $B$ in $\mathrm{C}_{G}(u)$ where $u \in D$ and $l(b)=1$. Let $\left|\mathrm{N}_{G}(\langle u\rangle, b): \mathrm{C}_{G}(u)\right|=p^{s} r$ with $s \geq 0$ and $p \nmid r$. Then

$$
k_{0}(B) \leq \frac{|\langle u\rangle|+p^{s}\left(r^{2}-1\right)}{|\langle u\rangle| r} p^{d}
$$

where $d$ is the defect of $b$.

Proof. Setting $q:=|\langle u\rangle|$ we obtain $C=\left(p^{d} / q\right)$ in the situation of Theorem B. By Lemma 9, $k_{0}(\langle u\rangle \rtimes \mathcal{N})=\left(q+p^{s}\left(r^{2}-1\right)\right) / r$ and the claim follows with $W=(1)$.

The following result of Brauer cannot be seen in the framework of integral quadratic forms. It was a crucial ingredient in the proof of the $k(G V)$-Problem (see [19, Theorem 2.5d]).

Corollary 15 (Brauer [5, 5D]). Let $B$ be a p-block with defect d, and let $C$ be the Cartan matrix of a Brauer correspondent $b$ of $B$ in $\mathrm{C}_{G}(u)$ where $u \in \mathrm{Z}(D)$. Then $k(B) \leq l(b) / m \leq l(b) p^{d}$ where

$$
m:=\min \left\{x C^{-1} x^{\mathrm{t}}: x \in \mathbb{Z}^{l(b)} \backslash\{0\}\right\} .
$$

Proof. By the definition of $m$, the matrix $W:=\frac{1}{m} C^{-1}$ is integral positive definite. Theorem A gives $k(B) \leq \operatorname{tr}(W C)=l(b) / m$. For the second inequality we recall that the elementary divisors of $C$ divide $p^{d}$. Hence, $p^{d} C^{-1}$ has integral entries and $m \geq p^{-d}$.

In [16], we referred to the Cartan method and the inverse Cartan method when applying Corollary 13 and Corollary 15 respectively. Now we know that both methods are special cases of a single theorem. In fact, the following examples show that Theorem A is stronger than Corollary 13 and Corollary 15.

(i) Let $B$ be the principal 2-block of the affine semilinear group $G=\mathrm{A} \Gamma \mathrm{L}(1,8)$, and let $u=1$. Then

$$
C=\left(\begin{array}{lllll}
2 & \cdot & \cdot & 1 & 1 \\
\cdot & 2 & . & 1 & 1 \\
\cdot & \cdot & 2 & 1 & 1 \\
1 & 1 & 1 & 4 & 3 \\
1 & 1 & 1 & 3 & 4
\end{array}\right)
$$

and $m=\frac{1}{2}$ with the notation of Corollary 15 . This implies $k(B) \leq 10$. On the other hand, $q\left(x_{1}, \ldots, x_{5}\right)=x_{1}^{2}+\ldots+x_{5}^{2}+x_{1} x_{2}-x_{1} x_{5}-x_{2} x_{5}-x_{3} x_{5}-x_{4} x_{5}$ in Corollary 13 gives $k(B) \leq 8$ and in fact equality holds (cf. [10, p. 84]). 
(ii) Let $B$ be the principal 2-block of $G=A_{4} \times A_{4}$ where $A_{4}$ denotes the alternating group of degree 4 . Let $u=1$. Then

$$
C=\left(1+\delta_{i j}\right)_{i, j=1}^{3} \otimes\left(1+\delta_{i j}\right)_{i, j=1}^{3}
$$

and $m=9 / 16$ with the notation of Corollary 15. Hence, we obtain $k(B) \leq 16$ and equality holds. On the other hand, it has been shown in [15, Section 3] that there is no positive definite, integral quadratic form $q$ such that $k(B) \leq 16$ in Corollary 13 .

We give a final application where the Cartan matrix $C$ is known up to basic sets. It reveals an interesting symmetry in the formula.

Proposition 16. Let $B$ be a block of a finite group with abelian defect group $D$ and inertial quotient $E \leq \operatorname{Aut}(D)$. Suppose that $u \in D$ such that $D /\langle u\rangle$ is cyclic. Then

$$
k(B) \leq\left(\left|\mathrm{N}_{E}(\langle u\rangle) / \mathrm{C}_{E}(u)\right|+\frac{|\langle u\rangle|-1}{\left|\mathrm{~N}_{E}(\langle u\rangle) / \mathrm{C}_{E}(u)\right|}\right)\left(\left|\mathrm{C}_{E}(u)\right|+\frac{|D /\langle u\rangle|-1}{\left|\mathrm{C}_{E}(u)\right|}\right) \leq|D| .
$$

Proof. With the notation of Theorem A we have $\mathcal{N}=\mathrm{N}_{E}(\langle u\rangle) / \mathrm{C}_{E}(u)$. Moreover, $\bar{b}$ has defect group $D /\langle u\rangle$ and inertial quotient $\mathrm{C}_{E}(u)$. By Dade's theorem on blocks with cyclic defect groups, $l(b)=$ $\left|\mathrm{C}_{E}(u)\right|$ and $C=\left(m+\delta_{i j}\right)$ up to basic sets where $m:=(|D /\langle u\rangle|-1) / l(b)$ (see [16, Theorem 8.6]).

With $W=U_{l(b)}$ we obtain

$$
\begin{aligned}
k(B) & \leq\left(|\mathcal{N}|+\frac{\mid\langle u\rangle-1}{|\mathcal{N}|}\right) \operatorname{tr}(W C) \\
& =\left(\left|\mathrm{N}_{E}(\langle u\rangle) / \mathrm{C}_{E}(u)\right|+\frac{|\langle u\rangle|-1}{\left|\mathrm{~N}_{E}(\langle u\rangle) / \mathrm{C}_{E}(u)\right|}\right)\left(\left|\mathrm{C}_{E}(u)\right|+\frac{|D /\langle u\rangle|-1}{\left|\mathrm{C}_{E}(u)\right|}\right) .
\end{aligned}
$$

The first factor is at most $|\langle u\rangle|$ and the second factor is bounded by $|D /\langle u\rangle|$. This implies the second inequality.

In every example we have checked so far, Theorem A implies Brauer's $k(B)$-Conjecture.

\section{Acknowledgment}

I have been pursuing these formulas since my $\mathrm{PhD}$ in 2010 and it has always remained a challenge to prove the most general. The work on this paper was initiated in February 2018 when I received an invitation by Christine Bessenrodt to the representation theory days in Hanover. I thank her for this invitation. The paper was written in summer 2018 while I was an interim professor at the University of Jena. I like to thank the mathematical institute for the hospitality and also my sister's family for letting me stay at their place. Moreover, I appreciate some comments on algebraic number theory by Tommy Hofmann. The work is supported by the German Research Foundation (projects SA 2864/1-1 and SA 2864/3-1). 


\section{References}

[1] J. L. Alperin and M. Broué, Local methods in block theory, Ann. of Math. (2) 110 (1979), $143-157$.

[2] M. Aschbacher, R. Kessar and B. Oliver, Fusion systems in algebra and topology, London Mathematical Society Lecture Note Series, Vol. 391, Cambridge University Press, Cambridge, 2011.

[3] J. Brandt, A lower bound for the number of irreducible characters in a block, J. Algebra 74 (1982), 509-515.

[4] R. Brauer, On blocks of characters of groups of finite order. II, Proc. Nat. Acad. Sci. U.S.A. 32 (1946), 215-219.

[5] R. Brauer, On blocks and sections in finite groups. II, Amer. J. Math. 90 (1968), 895-925.

[6] R. Brauer and W. Feit, On the number of irreducible characters of finite groups in a given block, Proc. Nat. Acad. Sci. U.S.A. 45 (1959), 361-365.

[7] M. Broué, On characters of height zero, in: The Santa Cruz Conference on Finite Groups (Univ. California, Santa Cruz, Calif., 1979), 393-396, Proc. Sympos. Pure Math., Vol. 37, Amer. Math. Soc., Providence, RI, 1980.

[8] G. H. Hardy and E. M. Wright, An introduction to the theory of numbers, Sixth edition, Oxford University Press, Oxford, 2008.

[9] L. Héthelyi, B. Külshammer and B. Sambale, A note on Olsson's Conjecture, J. Algebra 398 (2014), 364-385.

[10] B. Külshammer and T. Wada, Some inequalities between invariants of blocks, Arch. Math. (Basel) 79 (2002), 81-86.

[11] G. Navarro and B. Sambale, A counterexample to Feit's Problem VIII on decomposition numbers, J. Algebra 477 (2017), 494-495.

[12] J. B. Olsson, Inequalities for block-theoretic invariants, in: Representations of algebras (Puebla, 1980), 270-284, Lecture Notes in Math., Vol. 903, Springer-Verlag, Berlin, 1981.

[13] G. R. Robinson, On the number of characters in a block, J. Algebra 138 (1991), 515-521, Corrigendum: J. Algebra 144 (1991), 266-267.

[14] B. Sambale, Cartan matrices and Brauer's k(B)-conjecture, J. Algebra 331 (2011), 416-427.

[15] B. Sambale, Cartan matrices and Brauer's k(B)-conjecture II, J. Algebra 337 (2011), 345-362.

[16] B. Sambale, Blocks of finite groups and their invariants, Springer Lecture Notes in Math., Vol. 2127, Springer-Verlag, Cham, 2014.

[17] B. Sambale, Cartan matrices and Brauer's k(B)-Conjecture III, Manuscripta Math. 146 (2015), 505-518.

[18] B. Sambale, Refinements of the orthogonality relations for blocks, Algebr. Represent. Theory 20 (2017), 1109-1131.

[19] P. Schmid, Some remarks on the $k(G V)$ theorem, J. Group Theory 8 (2005), 589-604.

[20] T. Wada, A lower bound of the Perron-Frobenius eigenvalue of the Cartan matrix of a finite group, Arch. Math. (Basel) 73 (1999), 407-413. 